\title{
Pros and cons of microwave imaging techniques for the reconstruction of velocity profiles of cylindrical targets in axial motion
}

\author{
Massimo Brignone \#, Matteo Pastorino \#, Mirco Raffetto \#, Andrea Randazzo \# \\ \#Dept. of Electrical, Electronic, Telecommunication Engineering, and Naval \\ Architecture (DITEN), University of Genoa, Via Opera Pia 11a, 16145, Genoa, Italy
}

\begin{abstract}
In this contribution the strengths and the weaknesses of microwave imaging techniques for the reconstruction of dielectric and velocity profiles of inhomogeneous cylinders in non-uniform axial motion are presented. Some numerical results are presented to support the main conclusions.
\end{abstract}

\section{INTRODUCTION}

The reconstruction of the dielectric and velocity profiles in pipelines is a topic of great practical interest. Pneumatic or oil pipelines are just a few examples [1], [2]. Such a reconstruction can be based on acoustic or electromagnetic waves. Acoustic sensors could be in trouble when they are exploited to recover the velocity profile of media in motion in pipelines [1]. For this reason, most of the techniques developed in the last decades are based on electromagnetic phenomena in different frequency bands, ranging from low [2] to optical frequencies [1].

Recently, our research group has proposed additional electromagnetic approaches [3], based on microwave inverse scattering techniques [4]. Such techniques have been proved to possess excellent properties in traditional inverse scattering problems [4], but to the best of authors knowledge, they have never been used before to solve inverse scattering problems involving the reconstruction of velocity profiles.

The most trivial application of inverse scattering techniques can provide satisfactory results when the motion is characterized by huge axial velocities [3], [5], [6]. For nonrelativistic velocities a two-step algorithm provides much better performances than the traditional one-step extension of any microwave inverse scattering approach [3].

In this contribution, the strengths and the weaknesses of the proposed microwave inverse scattering techniques are investigated and some comparisons are performed.

\section{THE PROPOSED APPROACHES IN SHORT}

In microwave inverse scattering techniques, information about dielectric and velocity profiles of cylindrical targets in axial motion can be recovered from the measurements of the axial components of the electromagnetic scattered field, $E^{s}$ and $H^{s}$. In fact, such components depend on $\varepsilon_{r}, \mu_{r}$ and $\beta=\frac{v}{c_{0}}$ (here $v$ is the velocity along the axis of the pipeline and $c_{0}$ is the speed of light in vacuum, as usual), on the position of the transmitting antenna $x_{s}$ and on the position of the receiving sensor $x_{m}$. Therefore we can use the notation $E^{s}\left(x_{s}, x_{m} ; \mu_{r}, \varepsilon_{r}, \beta\right)$ and $H^{s}\left(x_{s}, x_{m} ; \mu_{r}, \varepsilon_{r}, \beta\right)$.

The most trivial application of a microwave inverse scattering technique tries to recover the fields of the constitutive parameters and the velocity profile by solving an optimization problem. More precisely, in the presence of $S$ transmitting antennas and $M$ receiving sensors, if the measured axial components of the electromagnetic field are denoted by $E_{m}^{s}\left(x_{s}, x_{m} ; \mu_{r}, \varepsilon_{r}, \beta\right)$ and $H_{m}^{s}\left(x_{s}, x_{m} ; \mu_{r}, \varepsilon_{r}, \beta\right)$, $s=1, \ldots, S, m=1, \ldots, M$, then one has to find $\mu_{r}^{t}, \varepsilon_{r}^{t}$ and $\beta^{t}$ that minimize the cost function

$$
f\left(\mu_{r}^{t}, \varepsilon_{r}^{t}, \beta^{t}\right)=g\left(\mu_{r}^{t}, \varepsilon_{r}^{t}, \beta^{t}\right)+h\left(\mu_{r}^{t}, \varepsilon_{r}^{t}, \beta^{t}\right),
$$

where

$$
\frac{g\left(\mu_{r}^{t}, \varepsilon_{r}^{t}, \beta^{t}\right)=}{\sum_{s, m}\left|E^{s}\left(x_{s}, x_{m} ; \mu_{r}^{t}, \varepsilon_{r}^{t}, \beta^{t}\right)-E_{m}^{s}\left(x_{s}, x_{m} ; \mu_{r}, \varepsilon_{r}, \beta\right)\right|^{2}}
$$

and $h\left(\mu_{r}^{t}, \varepsilon_{r}^{t}, \beta^{t}\right)$ is the same as $g\left(\mu_{r}^{t}, \varepsilon_{r}^{t}, \beta^{t}\right)$ but with $H$ in place of $E$. Another possible approach is based on a two-step procedure. In the first step one has to find $\mu_{r, 0}^{t}$ and $\varepsilon_{r, 0}^{t}$ that minimize $g\left(\mu_{r, 0}^{t}, \varepsilon_{r, 0}^{t}, 0\right)$ (respectively, $h\left(\mu_{r, 0}^{t}, \varepsilon_{r, 0}^{t}, 0\right)$ ), when a TM (respectively, TE) illuminating field is considered. In the second step, the recovered $\mu_{r, 0}^{t}$ and $\varepsilon_{r, 0}^{t}$ are exploited in order to find $\beta^{t}$ that minimizes $h\left(\mu_{r, 0}^{t}, \varepsilon_{r, 0}^{t}, \beta_{t}\right)$ (respectively, $\left.g\left(\mu_{r, 0}^{t}, \varepsilon_{r, 0}^{t}, \beta_{t}\right)\right)$. The main idea behind this approach is that the reconstruction of the constitutive parameters could be retained to be almost independent of the axial velocity.

\section{PROS AND CONS OF THE PROPOSED APPROACHES}

Inverse scattering approaches have some very well known features, which are retained for the applications here considered. In particular, among the good qualities of such approaches, one can observe that they do not require any change in the pipeline. For example, there is no need for entry and exit windows, which are required by other microwave or optical techniques [1]. Another good feature of the proposed approaches is related to the fact that they do not require to place sensors all around the pipe [7]. The illuminating and the receiving antennas can be placed on a single side, when the 
pipelines are not directly accessible. Finally, the approaches are potentially able to provide a complete description of the profiles of interest.

Among the weaknesses of the proposed approaches, one has usually to consider the lack of real time reconstructions of the profiles of interest. Moreover, in the first of the considered approaches, the unknown profiles are treated without any reference to their physical meaning and in [3] it has been shown that good results can be achieved only for large values of $\beta$. On the contrary, when the values of $\beta$ are known to be sufficiently small (i.e. $\max \beta<0.01$, see [3]) the twostep procedure provides much better and satisfactory results [3]. However, for very low axial velocities, it has been shown that it is necessary to have an excellent polarization purity of the transmitting antennas and an extremely good crosspolarization isolation for the receiving ones [7]. For example, as a rough estimate, a flow with an average normalized axial velocity $\beta$ of $10^{-r}, r \in \mathbb{N}$, cannot be recovered if the sensors do not guarantee a cross-polarization isolation of at least $20 r \mathrm{~dB}$ [7]. This kind of precision requirement on the instrumentation is not unusual, however. For example, in all techniques based on the Doppler-shift principle, the frequency shift (with respect to the signal frequency) to be appreciated is proportional to $\beta$.

\section{NUMERICAL RESULTS AND DISCUSSION}

Some negative results on the capabilities of the two microwave inverse scattering approaches are easily obtained.

For example, the first approach, based on a single step, is in trouble when all cylinders involved in the problem of interest move with small normalized values of the axial velocity. This is related to the fact that all unknown fields are considered without any distinction while one of the two axial components of the scattered field could be related to one of the unknown fields in a really weak way. In particular, even in the presence of a single cylinder made up of a few layers, when $\max \beta<$ $10^{-4}$ (which, by the way, corresponds to a huge velocity of $\simeq 30$ kilometers per second), the errors on the reconstruction of $\beta$ could be of the same order of $\beta$ itself [3].

By its very definition, the two-step approach, which neglects the movement in the first of its two reconstruction processes, is in trouble in the presence of relativistic velocities [3]. This limitation is capable of affecting the performances of both the reconstructions, the one related to the constitutive parameters and that related to the axial speed.

More elusive is the limitation of the two-step approach in relation to the cross-polarization properties of the sensors adopted. This type of limitation was investigated in [7] and is in any case only related to the second step of the procedure, devoted to the reconstruction of the axial velocity profile. The first step is simply not affected by this type of considerations, the movement being a priori neglected in this stage of the procedure (devoted to the reconstruction of $\varepsilon_{r}$ and $\mu_{r}$ ).

Numerical simulations show that the reliable reconstruction of the $\beta$ values even in the presence of a single homogeneous circular cylinder moving with a uniform axial speed cannot be achieved when $\beta$ is smaller than the cross-polarization isolation of the sensors adopted.

Thus, the two-step approach, in the presence of a given set of sensors, is known in advance to have a well defined range of $\beta$ values other which the reconstruction process of the axial velocity can provide reliable results. For example, for a cross-polarization isolation of $80 \mathrm{~dB}$ we get a range of $\beta$ approximately given by $\left[10^{-4}, 10^{-2}\right]$ [7]. With these sensors, we have considered the reconstruction of a three layer circular cylinder. The relative permittivity of each layer was assumed to get a value equal to 2 or 4 , for a set of 8 different dielectric configurations. All layers move with the same axial velocity $\beta \in\left\{10^{-8}, 10^{-7}, 10^{-6}, 10^{-5}, 10^{-4}, 10^{-3}, 10^{-2}\right\}$. By using ACO as an optimization technique [4], we get satisfactory results in terms of reconstructions of the relative permittivity values, with relative errors below $20 \%$ in all cases, for all considered values of $\beta$. The same optimization technique is not able to give the same quality of results in terms of reconstruction of (the constant) $\beta$. When $\beta$ is equal to or smaller than $10^{-5}$ the errors are, on average, well above $100 \%$. For higher $\beta$ values the performances improve very quickly and are reliable when $\beta$ is equal to or larger than $10^{-3}$, with average errors of a few percent and a small standard deviation.

These additional results confirm the reliability of the twostep approach, when it is exploited taking account of the well known a-priori limitations and of the performances of the sensors adopted in terms of cross-polarization isolation.

\section{CONCLUSION}

Microwave inverse scattering techniques have recently been introduced as potential alternative approaches to the reconstruction of dielectric and velocity profiles of cylinders in axial motion. Their theoretical and practical advantages and disadvantages are discussed. Some numerical results are presented to point out the main features of these novel approaches.

\section{REFERENCES}

[1] Y. Yan, "Mass flow measurement of bulk solids in pneumatic pipelines," Measurement Science and Technology, vol. 7, no. 12, p. 1687.

[2] W. Q. Yang, A. L. Stott, M. S. Beck, and C. G. Xie, "Development of capacitance tomographic imaging systems for oil pipeline measurements," International Journal of Multiphase Flow, vol. 22, no. 1001, pp. 144-145, 1996.

[3] M. Pastorino, M. Raffetto, and A. Randazzo, "Electromagnetic inverse scattering of axially moving cylindrical targets," IEEE Transactions on Geoscience and Remote Sensing, vol. 53, no. 3, pp. 1452-1462, March 2015.

[4] M. Pastorino, Microwave Imaging. New York: Wiley, 2010.

[5] M. Pastorino and M. Raffetto, "Scattering of electromagnetic waves from a multilayer elliptic cylinder moving in the axial direction," IEEE Transactions on Antennas and Propagation, vol. 61, no. 9, pp. 47414753, September 2013.

[6] M. Brignone and M. Raffetto, "Well posedness and finite element approximability of two-dimensional time-harmonic electromagnetic problems involving non-conducting moving objects with stationary boundaries,' ESAIM: Mathematical Modelling and Numerical Analysis, vol. 49, no. 4, pp. 1157-1192, July-August 2015.

[7] M. Brignone, G. L. Gragnani, M. Pastorino, M. Raffetto, and A. Randazzo, "Noise limitations on the recovery of average values of velocity profiles in pipelines by simple imaging systems," IEEE Geoscience and Remote Sensing Letters, vol. 13, no. 9, pp. 1340-1344, 2016. 